\title{
Influential Node Identification in Command and Control Networks Based on Integral $k$-Shell
}

\author{
Yunming Wang $\mathbb{D}^{1}{ }^{1}$ Bo Chen $\mathbb{D}^{2},{ }^{2}$ Weidong $L i,{ }^{1}$ and Duoping Zhang ${ }^{3}$ \\ ${ }^{1}$ School of Electrical and Information Engineering, Dalian Jiaotong University, Liaoning 116028, China \\ ${ }^{2}$ College of Mechanical and Electronical Engineering, Lingnan Normal University, Zhanjiang 524048, China \\ ${ }^{3}$ Communication and Network Laboratory, Dalian University, Liaoning 116622, China
}

Correspondence should be addressed to Yunming Wang; wang19871128@126.com

Received 6 July 2019; Revised 27 September 2019; Accepted 9 October 2019; Published 30 October 2019

Academic Editor: Xianfu Lei

Copyright (c) 2019 Yunming Wang et al. This is an open access article distributed under the Creative Commons Attribution License, which permits unrestricted use, distribution, and reproduction in any medium, provided the original work is properly cited.

\begin{abstract}
Influential nodes act as a hub for information transmission in a command and control network. The identification of influential nodes in a network of this nature is a significant and challenging task; however, it is necessary if the invulnerability of the network is to be increased. The existing $k$-shell method is problematic in that it features a coarse sorting granularity and does not consider the local centrality of nodes. Thus, the degree of accuracy with which the influential nodes can be identified is relatively low. This motivates us to propose a method based on an integral $k$-shell to identify the influential nodes in a command and control network. This new method takes both the global and local information of nodes into account, introduces the historical $k$-shell and a 2 -order neighboring degree, and refines the $k$-shell decomposition process in a network. Simulation analysis is carried out from two perspectives: to determine the impact on network performance when influential nodes are removed and to obtain the correlation between the integral $k$-shell value and its propagation value. The simulation results show that the integral $k$-shell method, which employs an algorithm of lower complexity, accurately identifies the influence of those nodes with the same $k$-shell values. Furthermore, the method significantly improves the accuracy with which the influential nodes can be identified.
\end{abstract}

\section{Introduction}

As the hinge for the command and control system to transfer information and fight order, command and control network (C2 network) is the key to win the war [1-3]. With the constant improvement of battlefield information, the organizational structure of the $\mathrm{C} 2$ network is becoming more and more complex, and the interaction of information is more frequent [4]. The $\mathrm{C} 2$ network shows the characteristics of heterogeneous nodes, multilayer structure, and so on. Command and control networks, which have the typical complex network characteristics, have scale-free characteristics. These networks exhibit the characteristics of "robustness and fragility" [5-7]; consequently, the problem associated with their vulnerability is a challenge that needs to be addressed. If the influential nodes in a network are attacked and damaged, the network structure is severely compromised, thus reducing the invulnerability of the command and control network and affecting the operational capability of the command and control system $[8,9]$. Therefore, identifying the influential nodes of a command and control network is vital to ensure the reliable operation of a network and enhance the operational capability of the system, which has both important theoretical significance and practical value.

The methods used to identify the influential nodes in a complex network are mainly divided into two categories: system science analysis methods and social network analysis methods [10]. The basic concept of system science analysis methods is that "destructiveness is equal to importance"; that is, the influence of a node is equal to the degree of damage to the network after the deletion of that node. The main identification methods are the shortest path method, which identifies the influential nodes by evaluating the 
difference in the shortest path after the deletion of any one node; the spanning tree method, which identifies the influential nodes by evaluating the number of spanning trees after the deletion of any one node; and the node shrinkage method, which identifies the influential nodes from the contraction of the network after the deletion of any one node. These methods have prompted research to identify the influential nodes in complex networks. However, the computational complexity of these methods is generally high, and the network nodes need to be tentatively tested, which is difficult in practice [11]. The main concept of social network analysis is that "importance is equal to saliency," with the influential nodes being identified by gathering statistics on the static characteristic indices of a network [12]. These indexes include the degree centrality [13], betweenness centrality [14, 15], approach degree [16], eigenvector [17], PageRank algorithm [18, 19], HITS algorithm $[20,21]$, structural hole $[22,23]$, and $k$-shell decomposition method [24].

The degree centrality method identifies the influential nodes by counting the number of neighboring nodes. A higher number of neighboring nodes imply that a node is more significant and therefore has a greater influence. The identification of influential nodes based on their degree centrality is simple and intuitive and has low computational complexity. However, this method considers only the local information of a node such that the identification accuracy is low. Chen et al. [25, 26] proposed a new method for identifying influential nodes based on local information. Their ultimate goal was to improve the recognition precision of the degree centrality of influential nodes. This method was based on the 4-order neighboring information of nodes and had relatively high recognition accuracy and lower computational complexity. These two methods for influential node identification considered only the number of neighboring nodes and disregarded the location of the node within the network structure. Nodes that are located in the core of real networks may have larger influence but a smaller degree. The betweenness centrality [14] method identifies influential nodes by calculating the number of paths through a node. A higher number of paths correspond to a greater degree of influence. It assumes that information is transmitted along only the shortest path between any pair of nodes. The PageRank algorithm [18, 19] is based on a cumulative nomination and is mainly used for web-page ranking. When web page $\mathrm{A}$ has a link pointing to web page $\mathrm{B}$, web page $B$ accrues a certain score. The PageRank algorithm provides a ranking for web-page matching according to the information provided by users, and the algorithm complexity is low. When an isolated node or community exists on a web page, the ranking of the PageRank algorithm is not unique. The Hypertext-Induced Topic Search (HITS) algorithm $[20,21]$ determines the most relevant web pages for a search by using an iterative approach. This algorithm offers wide applicability and low computational complexity. Kitsak et al. [24] defined the importance of nodes according to their location within a network and proposed a $k$-shell decomposition method. Although this method has low computational complexity and has been widely accepted, the method is not suitable for application to tree, star, or scalefree networks. This is because the identification results are too coarse, making the influential node difficult to distinguish within a shell. Zeng and Zhang [27] proposed a method based on mixing degree decomposition (MDD), which considers the number of remaining and deleted neighboring nodes to improve the visibility of the node influence. However, the scientific determination of the adjustable parameter $\lambda$ was not explained. As a means of processing a large number of nodes with the same $k$-shell value, which the $k$-shell decomposition method is unable to distinguish, Liu et al. [28] considered the shortest distance between a target node and the node set with the highest $k$ core value. They proposed a method using no parameters, which they named the distance to network-core (DNC) method. This method ranks the influence of the nodes in terms of the distance from a node to the network core, which is defined as the node set with the highest $k$-shell value. The $k$-shell decomposition method could identify the most influential spreaders of a network and also assign some nodes with the same value regardless of their role in the spreading process. In the same way, Ren et al. [29] aimed to overcome the difficulty of identifying influential nodes with the same $k$ shell value. Their approach was to combine the information on the neighboring nodes to determine the influential nodes with a minimum $k$-shell value, without changing the $k$-shell decomposition method. Hou et al. [30] proposed a new method, named the degree-betweenness- $k$-shell (DBK) method, to analyze the influence of the important nodes by using the Euler formula to combine the indexes of degree, betweenness, and $k$-shell. This method is characterized by high computational complexity. Finally, the social network analysis method depends on the network topology, without considering the heterogeneity and hierarchy of the node. There is a certain sidedness to the exploration of influential nodes when relying on a single index such that it is not possible to fully reflect the characteristics of the networks. Subsequently, Helbing and Podobnik et al. [31, 32] highlighted that suitable system design and management can prevent undesirable cascade effects and promote favorable kinds of self-organization in the system. Perc et al. [33, 34] reviewed models that describe information cascades in complex networks, with an emphasis on the role and consequences of node centrality. Mahdi also showed that some centrality measures, such as the degree and betweenness, are positively correlated with the spreading influence.

Research into influential node identification in a command and control network led to the introduction of the concept of a combat ring [35-37]. A combat network model for a weapons and equipment system based on a combat ring is established. The natural connectivity index is proposed as a measure of the survivability of a combat network. The measurement model has high sensitivity and low computational complexity. A network robustness measure based on a maximal connected subgraph was proposed. The measure enabled the robustness of networks to be evaluated for all kinds of attacks, including random attacks, degree rank attacks, and betweenness rank attacks [38]. The weighted 
algebraic connectivity was employed to analyze the robustness of the network structure when faced with an uncertain disturbance [39]. This work concluded that a highdensity node posse would induce more connections and greater usage, leading to increased unreliability. The entropy of the network structure was used to measure the order and stability of the supply chain system, which could be used to analyze the influence of the network scale and node connection probability on the stability of the system structure [40]. Note that research into the invulnerability of C2 networks is still in the early stages. The invulnerability of a combat network was investigated based on the connectivity of the nodes and edges [41]. The average path length was employed to simulate the relationship between the network structure and the efficiency of the network operations on the combat unit. This study concluded that the network average distance was an effective index for measuring networkcentric warfare, where the essential concept was to employ traditional measures in complex networks. However, no special attention was paid to the applicability of a C2 network. From this perspective, the Perron-Frobenius eigenvalue (PFE) of the adjacency matrix is often used to measure the network performance of an information age combat model (IACM), as introduced by Cares [42]. Although this idea has been proposed, the specific application of an IACM has not been adequately investigated. This means that the PFE and other problems have also not been validated, neither by theoretical derivation nor by experimental verification. Subsequent research $[43,44]$ was carried out based on Cares's IACM theoretical model, whereby a simulation was also conducted based on NetLogo. This study first validated the PFE as an evaluation index for measuring the operational effectiveness of a network. The simulation experiments incurred various limitations. For example, decision nodes were not connected to the network, the network scale was too small, and the difference in the capability of a node itself was not adequately considered. The theory of "structural holes" to identify the influential nodes in complex networks was proposed [22]. This method used local information to calculate the constraints of structural holes with certain limitations. The hierarchical structure of a command and control network was considered, the concept of hierarchical flow betweenness was defined, and the constraint coefficient of the structural holes was calculated [23]. Although the algorithm was computationally efficient, it had poor versatility.

Many methods exist for identifying the influential nodes in complex networks; however, their application to a command and control network is still in its infancy, given its complex network characteristics such as the hierarchical structure, heterogeneous nodes, local-area collaboration, and large network scale. The huge number of nodes and changeable network topology in a command and control network make it difficult to apply existing methods to identify influential nodes in networks with high real-time requirements. In addition, a command and control network comprises a large number of leaf nodes. The degree of these nodes is 1 and the betweenness is 0 . The use of existing methods to identify influential nodes is problematic in that the computational complexity and accuracy cannot be taken into account. Based on this, this paper proposes a method to identify influential nodes based on the integral $k$-shell. This method inherits the advantage of the $k$-shell decomposition method, i.e., its low computational complexity. The proposed method overcomes the problem that arises when a large number of nodes have the same $k$-shell value, i.e., that it is impossible to identify the influential nodes. Therefore, it greatly improves the recognition accuracy of the $k$-shell algorithm.

\section{Integral $k$-Shell Method}

The $k$-shell decomposition method is a classical influential node identification algorithm for application to complex networks. The principle of the algorithm is to shell the network layer and divide the nodes into different layers around the core. As the influence of the nodes in the central core increases, their importance also increases [45-47]. Although the complexity of the $k$-shell algorithm is low, the recognition accuracy is overly coarse-grained such that the influence of those nodes with the same $k$-shell value cannot be distinguished. To overcome the shortcomings of the $k$-shell decomposition method, an integral $k$-shell (IKS) decomposition method is proposed. This section first briefly introduces the principle of the $k$-shell decomposition method and then describes the IKS method in detail.

2.1. $k$-Shell Decomposition Method. Within a network, the $k$ shell decomposition method recursively shells those nodes with a degree that is less than or equal to $k$. The method considers the location of the nodes in the network and the aggregation characteristics of the other nodes, thus overcoming the limitations of the degree centrality method. Assuming that there are no isolated nodes with a degree of 0 in the network, those nodes with a degree of 1 are the least influential nodes in the network from the perspective of the degree index. Therefore, those nodes with a degree of 1 and their edges are deleted from the network first. After this deletion, new nodes with a degree of 1 appear in the network. These new nodes and their edges are then deleted, and the process is repeated until there are no new nodes with a degree of 1 within the network. At this point, all the deleted nodes form the first level, that is, the 1-shell, and the $k$-shell value of these nodes is 1 . The degree of each of the nodes remaining in the network is at least 2 . By repeating the above deletion operation, it is possible to acquire a second layer for which the $k$-shell value is equal to 2 , that is, the 2-shell. This process is continued until all the nodes in the network are assigned $k$-shell values. A diagram of the $k$-shell decomposition is shown in Figure 1. The nodes in the different circles belong to different cores, namely, the 1shell, 2-shell, and 3-shell, from the outside to the inside. The $k$-shell decomposition method has a low time complexity of only $o(m)$. This is extremely advantageous for large-scale network analysis; however, because a large number of nodes are assigned the same $k$-shell value, their 
influence cannot be identified, resulting in approximate partitioning results.

\subsection{Definition and Mathematical Description of Integral $k$ -} Shell. An analysis of the specific process of the $k$-shell decomposition method reveals the shortcomings described below.

First, by shelling the core, layer by layer, a large number of nodes are found to have the same $k$-shell value. As such, the influence of the nodes in the same shell cannot be determined, and the ranking results are too coarse-grained.

Second, the $k$-shell decomposition method only considers the residual degree of the current node; however, it does not consider the number of neighbors removed by the node. That is, although the method considers the global centrality of the node, it does not consider the local centrality of the node.

Third, the $k$-shell decomposition method is not suitable for networks without obvious aggregation or a large number of nodes with the same degree, such as tree networks, star networks, and BA scale-free networks. A command and control network exhibits obvious scale-free characteristics, retains the skeleton of a tree network, and has a large number of nodes with a degree of 1 , without any obvious aggregation characteristics. Therefore, although the $k$-shell decomposition method has low computational complexity, it cannot be directly applied to rank influential nodes in a command and control network.

This study therefore attempted to address these shortcomings of the $k$-shell decomposition method. Herein, we propose an integral $k$-shell (IKS) method to evaluate the influence of the nodes in a command and control network.

The $k$-shell decomposition method removes those nodes with the same shell values from the original network in different batches. In order to better distinguish the influential nodes among those with the same $k$-shell values and overcome the problem of the overly coarsegrained sorting results of the $k$-shell decomposition method, it is assumed that nodes removed later are more important than nodes removed earlier. When the IKS method is used to decompose a network, the $k$-shell value and batches are recorded at the time when each node is removed.

When using the $k$-shell method to decompose a network, it is necessary to consider both the number of $k$-shell values when the nodes are removed and the number of integral shelling times in each $k$-shell. When nodes in the same $k$ shell are removed from the network, a greater number of neighboring nodes would increase the shelling time. Therefore, the sum of the current $k$-shell values for the nodes and their historical $k$-shells can reflect the influence of nodes as they are removed from a network. This concept overcomes the problem of the $k$-shell method by considering only the global centrality of the nodes; however, it does not consider the local centrality of the nodes. According to reference [22], when calculating the influential nodes by considering the location and self-degree of nodes in the network, it is more accurate to get the ranking result of node

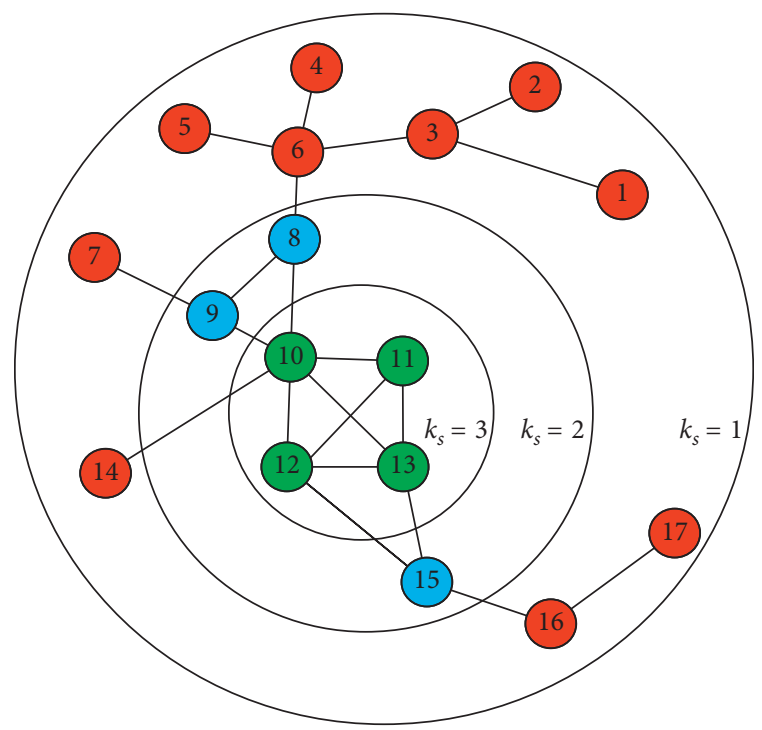

FIgURE 1: $k$-shell decomposition.

importance by calculating the 2-order neighboring degree, and the complexity is the lowest.

When analyzing the influence of nodes, it is necessary to consider the location and self-degree of the nodes within the network. The IKS method assumes that the influence of a node is also affected by its neighbors. If its neighbors are important, then the influence of that node is enhanced. This concept overcomes the problem of the $k$-shell method, i.e., that it is unsuitable for networks containing a large number of nodes of the same degree but no obvious aggregation.

Definition 1 ( $L$-order neighboring degree). For any node $v_{i}$ in a network, the number of neighboring nodes that can be reached in $N$ steps from the node $v_{i}$ is referred to as the $L$ order neighboring degree of the node $v_{i}$, which is denoted as $Q_{N}(i)$. Obviously, for any network, $G$ and $L$ are not larger than the network diameter, and $Q_{1}(i)=k_{i}$; that is, the 1order neighboring degree is equal to the degree of the nodes.

Definition 2 (historical $k$-shell). Based on the $k$-shell decomposition method, when any node $v_{i}$ is removed, the sum of the $k$-shell values corresponding to the previous shelling of the network is referred to as the historical $k$-shell of the node $v_{i}$.

Definition 3 (integral $k$-shell). For any node $v_{i}$ in a network, the sum of its 2-order neighboring degree and historical $k$ shell is termed the integral $k$-shell of the node $v_{i}$.

Given the basic concept of the IKS method, the integral $k$-shell index of each node $v_{i}$ is set to $i k s(i)$, which can be expressed as follows:

$$
i k s(i)=Q_{2}(i)+\sum_{j=1}^{m_{i}} k_{S}(i)
$$

where $Q_{2}(i)$ is the 2-order neighboring degree of the node $v_{i}$, $m_{i}$ is the number of times the network is shelled when the 
node $v_{i}$ is removed, and $k_{S}(j)$ represents the $k$-shell value when the network has been shelled $j$ times.

\section{Influential Node Identification in Command and Control Networks Based on Integral $k$-Shell}

A command and control network is characterized by its large scale, heterogeneous nodes, multiple interleaved links, and scale-free nature. At the same time, given the influence of a military organizational structure, command entities have strict subordinate command relationships in a command and control system. The relationships between the higher and lower entities in a command organization are characterized by a hierarchical topological structure in the command and control network [48]. The heterogeneity of the nodes indicates that there are relatively influential nodes in a command and control network, which must be protected to enhance the invulnerability of the network. The hierarchy of the structure indicates that there are a large number of leaf nodes with a degree of 1 and a betweenness of 0 in a command and control network. The complex characteristics of a command and control network determine that the general method used to identify influential nodes cannot be easily applied to this network. The integral $k$-shell method contains the historical $k$-shell value and the 2-order neighboring degree. The method also comprehensively takes the global and local information of the node into account to more accurately identify the influential nodes of the command and control network.

3.1. Algorithms for Influential Node Identification. Given the basic concept of the integral $k$-shell and the definition of the node $i k s(i)$, an algorithm for influential node identification based on the integral $k$-shell for a command and control network is proposed. The algorithm is based on the $k$-shell decomposition method, which takes into account the local and global centrality of nodes, and evaluates the influence of nodes from their $i k s(i)$ value. The integral $k$ shell algorithm is modified to enable it to be used for scalefree networks. The modified design considers the complexity and accuracy of the algorithm and introduces the 2 -order neighboring degree when calculating the $i k s(i)$ value of a node. The specific steps of the algorithm are as follows:

Step 1: calculate the $L$-order neighboring degree $L(i)$ of each node; mark the degree of each node as the initial $i k s(i)$ value, namely, $i k s(i)=L(i)$; and initialize $k_{S}=1$.

Step 2: use the $k$-shell decomposition method to construct the $k$-shell. First, remove the nodes in the outermost layer, for which the degree is $k_{S}$. Then, update the integral $k$-shell values for each node in the network such that $i k s(i)=i k s(i)+k_{S}$.

Step 3: remove those nodes with a degree of $k_{S}$ from the remaining network, which is newly created. Then, update the integral $k$-shell values of the nodes in the network again such that $i k s(i)=i k s(i)+k_{S}$.

Step 4: repeat Steps 2 and 3 to delete the new nodes for which the degree is $k_{S}$, and update the integral $k$-shell values of each node, until no nodes with a degree of $k_{S}$ remain in the network. The nodes removed up to this point constitute the $k_{S}-$ shell.

Step 5: update $k_{S}$ and $i k s(i)$ such that $k_{S}=k_{S}+1$ and $i k s(i)=i k s(i)+k_{S}$.

Step 6: count the number of remaining nodes in the network as $N r$. If $N r>0$, return to Step 2 and continue the $k$-shell decomposition until $N r=0$, at which point the algorithm terminates.

After decomposing the network according to the integral $k$-shell method, the $i k s(i)$ array indicates the influence of all the nodes. Then, the elements of the $i k s(i)$ array are ranked to determine the influence of the nodes in the command and control network. A higher $i k s(i)$ value corresponds to a node with greater influence.

The calculation of the 2-order neighboring degree of a node needs to traverse all the nodes in a network. The neighboring node set $N i_{-}$Set of the node $v_{i}$ is summed, and then the neighboring nodes of each node in the neighboring node set $\mathrm{Ni}$ Set are found. After removing the duplicate nodes and the node $v_{i}$, the set becomes the 2-order neighboring set $N i_{-} Z$ uhe $(i)$ of the node $v_{i}$. The number of nodes in the set is the number of 2-order neighbors of the node $v_{i}$.

The above analysis indicates that when calculating the 2-order neighboring degree of a node, the first step is to find the neighboring node of each node, that is, to calculate the degree of that node. For any node $v_{i}$, it is necessary to find those nodes that are directly connected to node $v_{i}$ from $N-1$ nodes except the node $v_{i}$ in the set $\mathrm{Ni}$ Set. The complexity of the algorithm is as follows:

$$
f_{1}=o(N-1)
$$

In the second step, for any point $v_{j}$ in the set $N i_{-}$Set, it remains necessary to find those nodes that are directly connected to the node $v_{j}$ from $N-1$ nodes, except the node $v_{j}$ in the set $N j_{-}$Set. The complexity remains $o(N-1)$. Assuming that the degree of the node $v_{i}$ is $k_{i}$, the computational complexity of the 2 -order neighboring degree of the node $v_{i}$ is

$$
f_{2}=f_{1}+k_{i} \cdot o(N-1) \text {. }
$$

If the total number of nodes in the network is $N$, and the average degree is $\langle k\rangle$, then all the nodes in the network are traversed and the 2-order neighboring degree of each node is solved. The complexity of the algorithm is as follows:

$$
f=N \cdot f_{2}=o(N(N-1)+\langle k\rangle \cdot N(N-1)) \approx o\left(N^{2}\langle k\rangle\right) .
$$

The core of the influential node-ranking algorithm based on the integral $k$-shell continues to be $k$-shell 
decomposition. The complexity of the $k$-shell decomposition algorithm is $o(N)$. The complexity of the influential node-ranking algorithm based on the integral $k$ shell is the sum of the complexities of the 2-order neighboring algorithm and the $k$-shell decomposition algorithm. The complexity is as follows:

$$
f=o(N)+o\left(N^{2}\langle k\rangle\right) \approx o\left(N^{2}\langle k\rangle\right) .
$$

Therefore, the complexity of the influential noderanking algorithm based on the integral $k$-shell is $o\left(N^{2}\langle k\rangle\right)$.

The classical $k$-shell decomposition method does not involve calculation of the distance between the nodes, but rather decomposes the network, layer by layer. The complexity of the algorithm is $o(N)$. The $k$-shell value of each shell increases by 1 . However, this causes low identification accuracy and results in poor applicability. Compared with the $k$-shell algorithm, the MDD algorithm [27] is an obvious improvement, in that the network can be divided into a greater number of cores, in which case the computational complexity remains as $o(N)$. For different networks, however, the value of $\lambda$ cannot be determined. The DNC algorithm [28] involves the calculation of the distance between the nodes, and its complexity is $o\left(N^{2}\right)$. However, although it can efficiently identify the influential nodes of a network, it cannot be applied to a command and control network with a hierarchical structure.

The distance between the nodes in the same layer of a command and control network and the maximum $k$-shell of the network is the same. The DBK algorithm [30] is closely correlated with the betweenness centrality, and it can efficiently identify the influential nodes of a network. However, the computational complexity is very high, similar to that of the betweenness centrality, at $o\left(N^{3}\right)$, making it difficult to apply to a command and control network. The computational complexities of the $k$-shell decomposition, MDD algorithm, DNC algorithm, DBK algorithm, degree centrality (DC), betweenness centrality (BC), and IKS algorithm, described herein, are listed in Table 1.

The identification of influential nodes in a command and control network based on the IKS algorithm does not change the process of $k$-shell decomposition. Instead, it improves the criteria for determining the influential nodes such that the influential nodes in the same shell can be more easily distinguished, thus inheriting the advantages of the $k$-shell decomposition method. Therefore, the IKS algorithm is suitable for command and control networks with a large number of nodes, frequent changes in the network structure, and high real-time requirements.

3.2. Example Analysis. The specific calculation process of the IKS algorithm is further analyzed by examining the $i k s(i)$ values of the nodes shown in Figure 1 as an example. In the example, the $i k s(10)$ values of the node $v_{10}$ are calculated. The specific calculation process is as follows.

First, the 2-order neighboring degree of the node $v_{10}$ is calculated. The set of neighboring nodes of the node $v_{10}$ is $N i$ Set $=\{8,9,11,12,13,14\}$. The neighboring nodes of nodes $8,9,11,12,13$, and 14 are $\{6,9,10\},\{7,8,10\},\{10,12$, $13\},\{10,11,13,15\},\{10,11,12,15\},\{10,11,12,15\}$, and $\{10\}$,
TABLE 1: Computational complexities.

\begin{tabular}{lc}
\hline Method & Complexity \\
\hline$k$-shell & $o(N)$ \\
MDD & $o(N)$ \\
DNC & $o\left(N^{2}\right)$ \\
DBK & $o\left(N^{3}\right)$ \\
DC & $o(N)$ \\
BC & $o\left(N^{3}\right)$ \\
IKS & $o\left(N^{2}\langle k\rangle\right)$ \\
\hline
\end{tabular}

respectively. Therefore, the set of 2-order neighboring nodes of the node $v_{10}$ is Ni_Zuhe $(10)=N i$ Set $\cup\{6,9,10\} \cup\{7,8,10\} \cup$ $\{10,12,13\} \cup\{10,11,13,15\} \cup\{10,11,12,15\} \cup\{10\}-\{10\}=\{6,7$, $8,9,11,12,13,14,15\}$, and the number of nodes in this set is 9 ; hence, the 2-order neighboring degree $N(10)$ of the node $v_{10}$ is 9 .

Second, the $k$-shell algorithm is used to decompose the network. All the nodes shelled from the network are listed in Table 2.

Table 2 indicates that when the $k$-shell decomposition network is used for the fifth decomposition, the node $v_{10}$ is removed such that $m_{10}=5$ and $\sum_{j=1}^{m_{10}} k_{S}(j)=1+1+1+2+$ $3=8$; thus, the $i k s(10)$ value of the node $v_{10}$ is

$$
i k s(10)=Q_{2}(10)+\sum_{j=1}^{m_{10}} k_{S}(j)=9+8=17 \text {. }
$$

According to the above-mentioned flow of the IKS algorithm, each node has a corresponding $i k s(i)$ value and $k$ shell, and the calculation process is the same as $i k s$ (10) IKS. The $i k s(i)$ values and $k$-shells of each node shown in Figure 1 are listed in Table 3.

In comparison, when using the $k$-shell decomposition method, all the nodes in the network shown in Figure 1 are divided into three layers. This ensures that the differences between the influential nodes are very small, and the ranking results are very coarse-grained. The IKS algorithm can clearly identify the influences of the nodes. Therefore, relative to the $k$-shell decomposition method, the IKS algorithm is more effective at identifying the influence of a given $k$-shell node.

\section{Simulation Analysis}

A typical command and control network model is established to verify the effectiveness and feasibility of the influential node identification method based on the IKS algorithm proposed in this paper. The topology of the command and control network is shown in Figure 2. The number of nodes $N$ is 453 , including 85 command nodes (red circles), 256 fire attack nodes (blue triangles), and 112 perception nodes (green squares). The command level $D$ is 5 , and the span $S$ is 4 .

This section presents the validation of the efficacy and generality of the IKS method from two aspects: the impact of the network performance when the influential nodes are removed and the correlation between the $i k s$ value and its propagation value. First, the network efficiency is taken as 
TABLE 2: Decomposing a network with the $k$-shell algorithm.

\begin{tabular}{lccc}
\hline Number of shellings $(j)$ & $k$-shell value & $k$-shell $(j)$ value & Shelled nodes from the network \\
\hline 1 & 1 & 1 & $1,2,4,5,7,14,17$ \\
2 & & 1 & 3,16 \\
3 & 2 & 2 & $6,9,15$ \\
4 & 3 & 3 & $10,11,12,13$ \\
\hline
\end{tabular}

TABLE 3: $i k s(i)$ values and $k$-shells of each node shown in Figure 1.

\begin{tabular}{lcc}
\hline Node label & $i k s(i)$ & $k_{s}-$ shell \\
\hline 1 & 4 & 1 \\
2 & 4 & 1 \\
3 & 8 & 1 \\
4 & 5 & 1 \\
5 & 5 & 1 \\
6 & 11 & 1 \\
7 & 4 & 1 \\
8 & 16 & 2 \\
9 & 13 & 2 \\
10 & 17 & 3 \\
11 & 15 & 3 \\
12 & 16 & 3 \\
13 & 16 & 3 \\
14 & 7 & 1 \\
15 & 11 & 2 \\
16 & 6 & 1 \\
17 & 3 & 1 \\
\hline
\end{tabular}

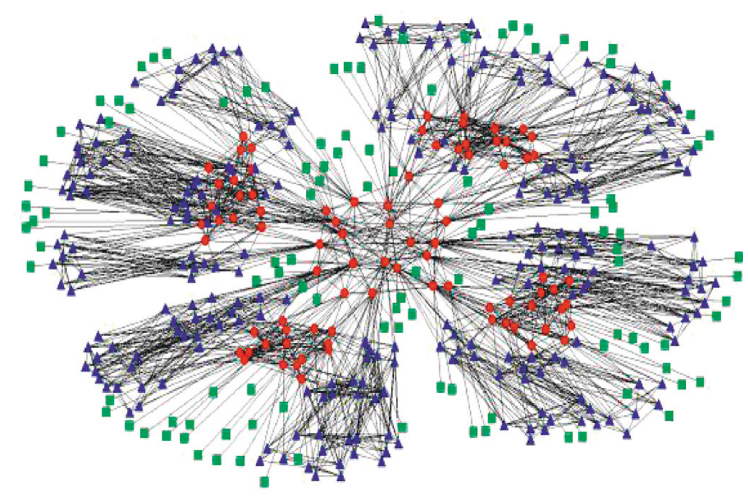

Figure 2: Command and control network model.

a measure, and node deletion is used to analyze the impact of the influential node removal on the network performance; second, the SIR propagation model is used to study the propagation ability of the influential nodes. Subsequently, Kendall's tau coefficient is used to analyze the correlation between the node influence values obtained by the various algorithms and the potential propagation abilities of the nodes. This approach makes it possible to verify the feasibility and accuracy of the IKS method.

4.1. Impact of Influential Node Removal on Network Performance. First, the $k$-shell, MDD, DNC, DBK, degree centrality (DC), betweenness centrality (BC), and IKS
TABle 4: Comparison of seven methods used to rank influential nodes.

\begin{tabular}{lccccccc}
\hline & \multicolumn{8}{c}{ Method } \\
No. & $k$-shell & MDD & DNC & DBK & DC & BC & IKS \\
\hline 1 & 2 & 10 & 37 & 10 & 10 & 7 & 10 \\
2 & 8 & 11 & 43 & 11 & 11 & 4 & 11 \\
3 & 10 & 7 & 46 & 9 & 9 & 13 & 4 \\
4 & 32 & 8 & 47 & 7 & 15 & 11 & 8 \\
5 & 33 & 14 & 2 & 5 & 37 & 14 & 9 \\
6 & 36 & 9 & 41 & 14 & 4 & 10 & 7 \\
7 & 37 & 4 & 42 & 8 & 7 & 9 & 37 \\
8 & 41 & 15 & 8 & 13 & 8 & 15 & 46 \\
9 & 42 & 37 & 10 & 15 & 14 & 8 & 14 \\
10 & 43 & 13 & 32 & 12 & 18 & 6 & 47 \\
\hline
\end{tabular}

methods were used to measure the influence of the nodes in a command and control network. The first 10 nodes in the network were ranked according to their influence, as shown in Table 4.

The results in Table 4 indicate that the ranking results obtained with the proposed IKS method are close to those of the $\mathrm{DBK}$ and $\mathrm{BC}$ methods for a command and control network and are quite different from those obtained with the $k$-shell method.

Second, based on the concept of "destructiveness being equal to importance," the influence of the nodes in a command and control network is ranked using the different methods described above. Based on the ranking results, one node is deleted from the initial network at a time, and different evaluation indicators are used to calculate the rate of decline in the network performance after the removal of each of the nodes. This cycle is repeated until all the nodes are removed such that the impact of each node on network performance can be calculated. Figures 3(a)-3(g) present the change in the network efficiency when nodes are deleted according to the $k$-shell, MDD, DNC, DBK, DC, BC, and IKS algorithms. Because of the huge number of nodes in a command and control network, only the relationship between the rate of decline in the network efficiency and the first 100 nodes in the influence ranking is shown in Figure 3.

As shown in Figure 3, the results of ranking the influence of the nodes using the seven different methods show that, after deleting nodes according to their order of influence, the network efficiency declines. This indicates that each method can basically distinguish the influence of most nodes. However, for the $k$-shell, MDD, and DNC methods, the rate of decline of the network efficiency does not strictly conform to the descending trend in the ranking results; furthermore, the fluctuations are large, indicating that the identification 


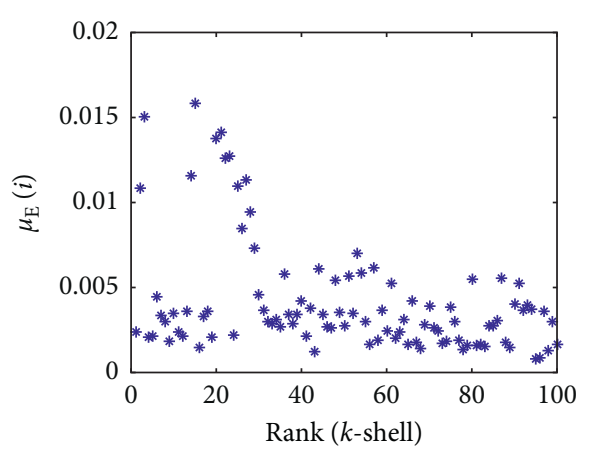

* $k$-shell

(a)

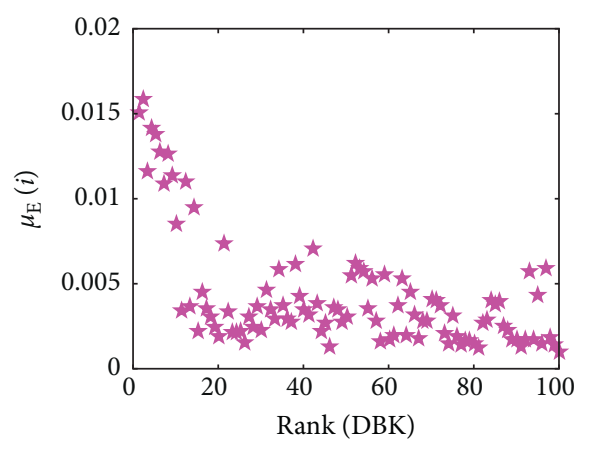

$\star$ DBK

(d)

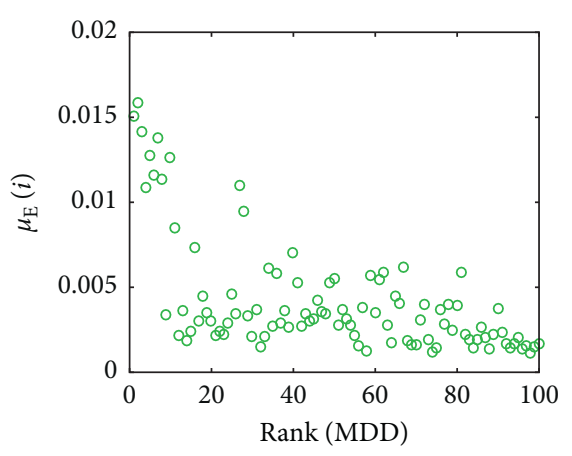

- MDD

(b)

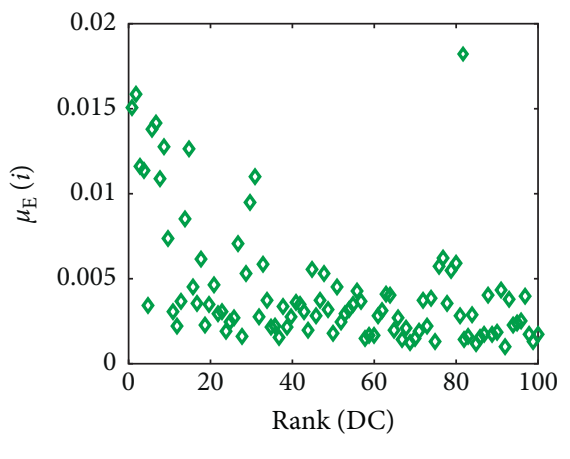

$\diamond \mathrm{DC}$

(e)

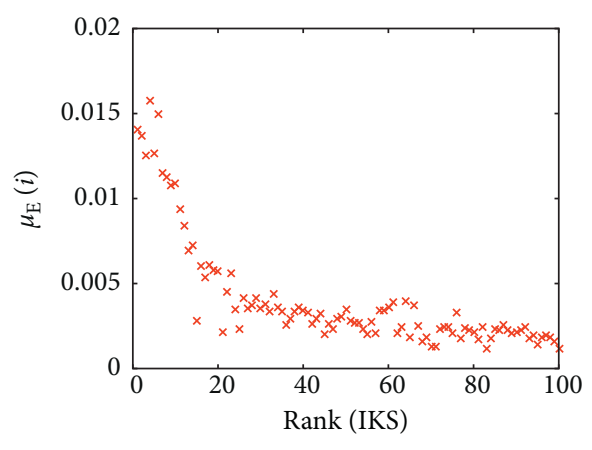

$\times$ IKS

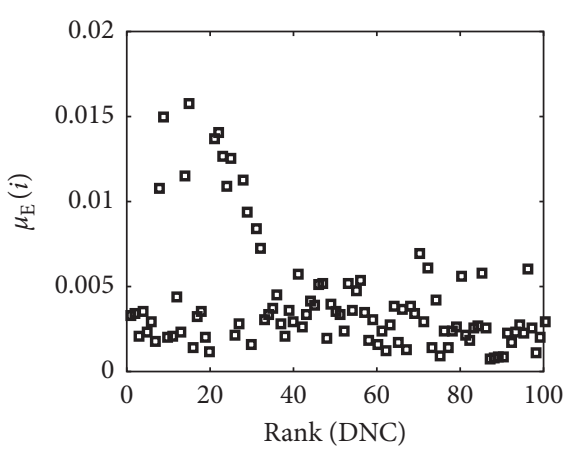

- DNC

(c)

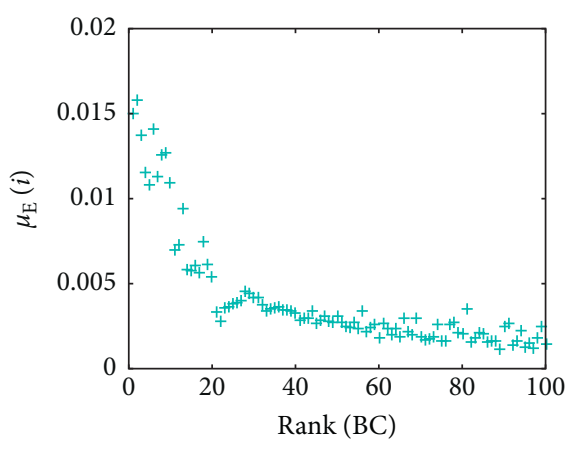

$+\mathrm{BC}$

\section{(g)}

FIGURE 3: Relationship between the rate of decline of network efficiency and importance rankings of nodes.

accuracy of the $k$-shell, MDD, DNC, and DC methods is low. When the proposed IKS and BC methods are used to rank the nodes, the rate at which the network efficiency decreases clearly most closely corresponds to the ranking result. This implies that these two methods can rank the nodes in the network most accurately and offer the best identification accuracy.

Additionally, the IKS method is also compared with other methods using Kendall's tau coefficient. The results, which are shown in Figures 4(a)-4(f), present the ranking comparison results of the IKS algorithm and the $k$-shell, MDD, DNC, DBK, DC, and BC algorithms, respectively. The dots in the graph represent the nodes of the network, and the color of the dots corresponds to the rate at which the network efficiency decreases after the nodes are removed. The abscissa corresponds to the influence value of the nodes calculated using the IKS method, that is, the $i k s(i)$ value of the node, whereas the ordinate corresponds to the influence of the nodes calculated using the other algorithms. Kendall's tau coefficient is $\tau$ and is used to measure the correlation between two sequences $X$ and Y. For any pair of nodes $\left(x_{i}, y_{i}\right)$ and $\left(x_{j}, y_{j}\right)$, if $x_{i}>x_{j}$ and $y_{i}>y_{j}$, or if $x_{i}<x_{j}$ and $y_{i}<y_{j}$, the pair of nodes is regarded as being relevant; if $x_{i}>x_{j}$ and $y_{i}<y_{j}$, or $x_{i}<x_{j}$ and $y_{i}>y_{j}$, the node pair is regarded as being irrelevant; if $y_{i}=y_{j}$, the correlation between the pair of nodes cannot be judged. The mathematical expression of Kendall's tau coefficient is as follows: 


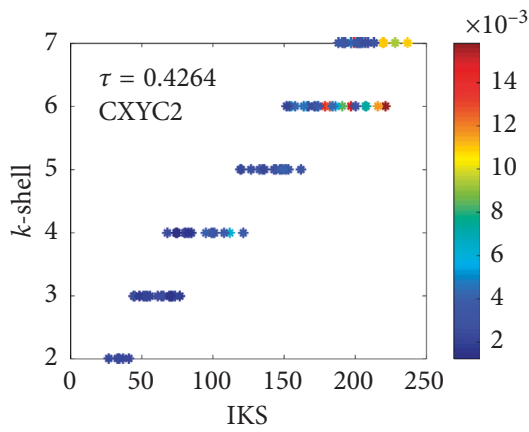

(a)

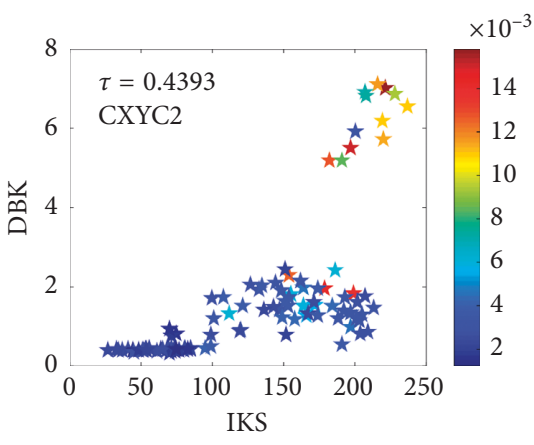

(d)

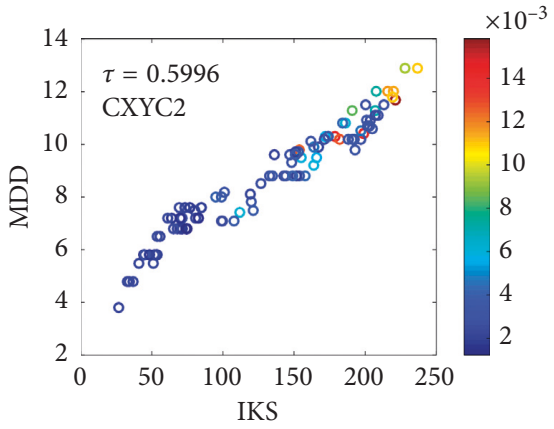

(b)

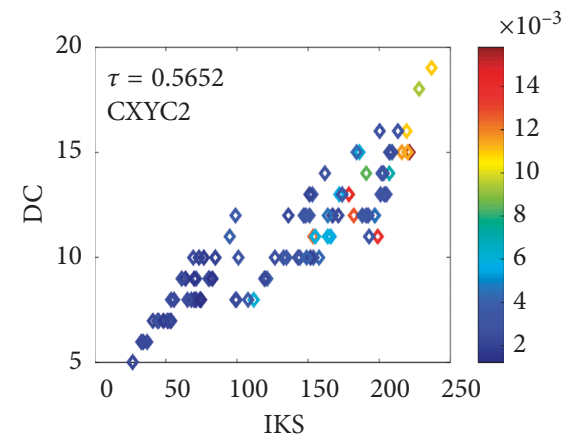

(e)

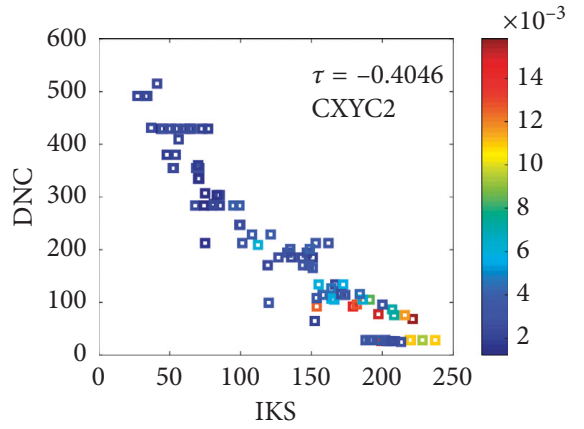

(c)

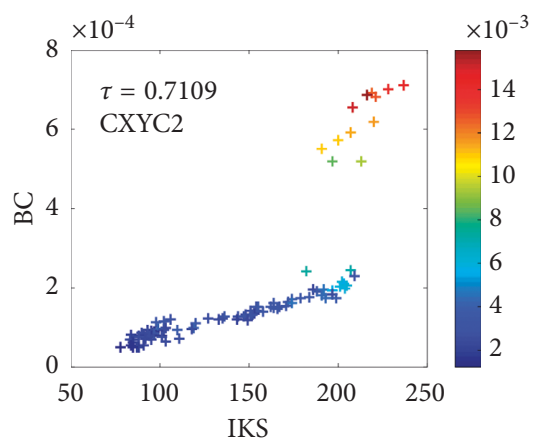

(f)

FIgURE 4: Relationship between the IKS method and other methods in terms of the ranking result.

$$
\tau=\frac{2\left(n_{1}-n_{2}\right)}{N(N-1)},
$$

where $N$ is the number of nodes, $n_{1}$ is the number of related node pairs in sequences $X$ and $Y$, and $n_{2}$ is the number of unrelated node pairs.

The analysis in Figure 4 shows that the ranking results of the IKS method proposed in this paper are positively correlated with those of the $k$-shell, MDD, DBK, DC, and BC methods. The correlation coefficient $\tau$ between the IKS and $\mathrm{BC}$ methods is the largest; that is, the ranking results obtained with these two methods are the most highly correlated. The IKS method is negatively correlated with the DNC method because with the DNC method, a smaller influence value corresponds to a greater node influence, which is different from that of other methods. The BC method is an existing influential node identification method that provides high-accuracy identification. The IKS method is most closely correlated with the BC method, which shows that the proposed method for influential node identification has high identification accuracy. In addition, the IKS and BC methods are superior to other methods according to the trend in the node color change. Generally, the IKS and BC methods are able to identify influential nodes more accurately in a command and control network. However, the IKS method has lower complexity than other methods. Therefore, considering the complexity and accuracy of the IKS method in combination, this method is superior in terms of identifying the influential nodes of a command and control network.

Next, the different methods are used to rank the influential nodes by sequentially deleting the first 45 nodes (the first $10 \%$ ) based on influence ranking in the network. The network efficiency, network connectivity coefficient, and maximum connected subgraph ratio are used to analyze the impact of the removal of influential nodes on the network, and the identification accuracies of the different methods are compared.

Figure 5 shows a plot of the rate at which the network efficiency $\mu_{\mathrm{E}}$ decreases as a function of the ratio of removed nodes $p$ after the removal of the influential nodes in a command and control network. The formula to calculate $\mu_{\mathrm{E}}$ is as follows:

$$
\mu_{\mathrm{E}}(i)=1-\frac{E_{i}^{\prime}}{E_{0}}
$$

where $E_{i}^{\prime}$ is the efficiency of the network after removing the nodes and $E_{0}$ is the initial network efficiency. As the value of $\mu_{\mathrm{E}}(i)$ increases, the network efficiency declines more drastically, indicating that the removal of the node $v(i)$ is more influential.

According to (8), the removal of influential nodes causes the network efficiency to decrease, whereas the rate of decline of the network efficiency increases. As can be 


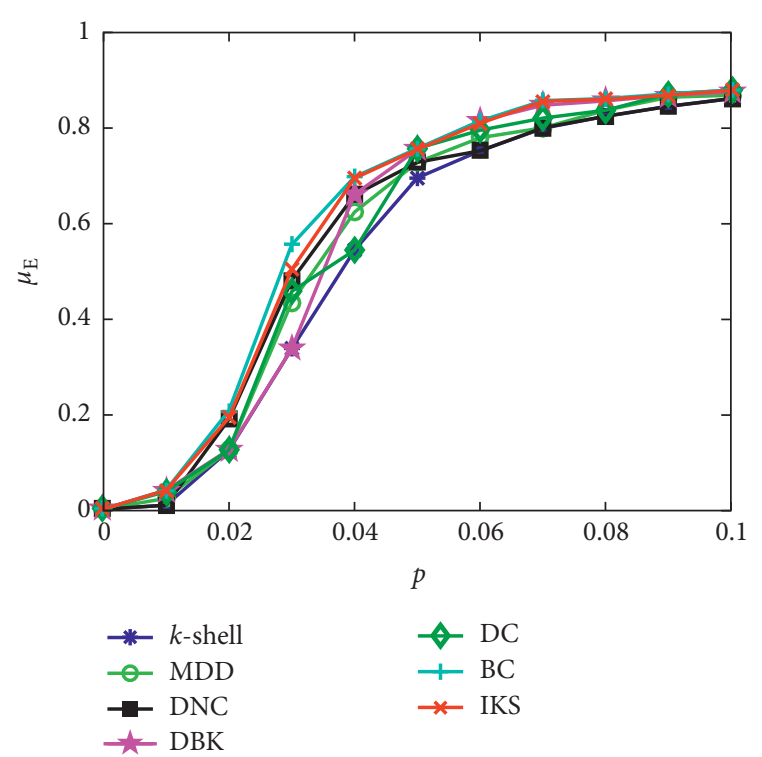

FIGURE 5: Relationship between the rate of decline of network efficiency and the ratio of removed nodes.

seen from Figure 5, macroscopically, an increase in the removal ratio of the influential nodes leads to an increase in the rate of decline of the network efficiency as more nodes are removed. Obviously, as the network efficiency falls, the connectivity deteriorates. Microscopically, the removal of the influential nodes first causes the curve for the IKS and BC methods to rise, and the network efficiency clearly decreases. This confirms the high accuracy of the ranking results of the IKS method, which is consistent with the simulation results shown in Figure 3.

The network connectivity coefficient examines the network performance from the perspective of network connectivity. Figure 6 shows the variation in the rate of descent of the network connectivity coefficient with the proportion $p$ of the removed nodes after the influential nodes of the command and control network are sequentially removed. The expressions defining the network connectivity coefficient $C$ and the rate of descent of the network connectivity coefficient $\mu_{\mathrm{C}}(i)$ are, respectively, as follows:

$$
\begin{aligned}
C & =\frac{1}{\omega \sum_{i=1}^{\omega}\left(N_{i} / N_{\text {init }}\right) l_{i}}, \\
\mu_{\mathrm{C}}(i) & =1-\frac{C_{i}^{\prime}}{C_{0}}
\end{aligned}
$$

where $\omega$ is the number of subnets in the network, $N_{\text {init }}$ is the total number of nodes in the network, and $N_{i}$ and $l_{i}$ are the number of nodes in the $i$ subnet and the average distance to the corresponding subnet, respectively. Furthermore, $C_{i}^{\prime}$ is the connectivity coefficient of the network after removing the nodes, and $C_{0}$ is the connectivity coefficient of the initial network. As the value of $\mu_{\mathrm{C}}(i)$ increases, the decline

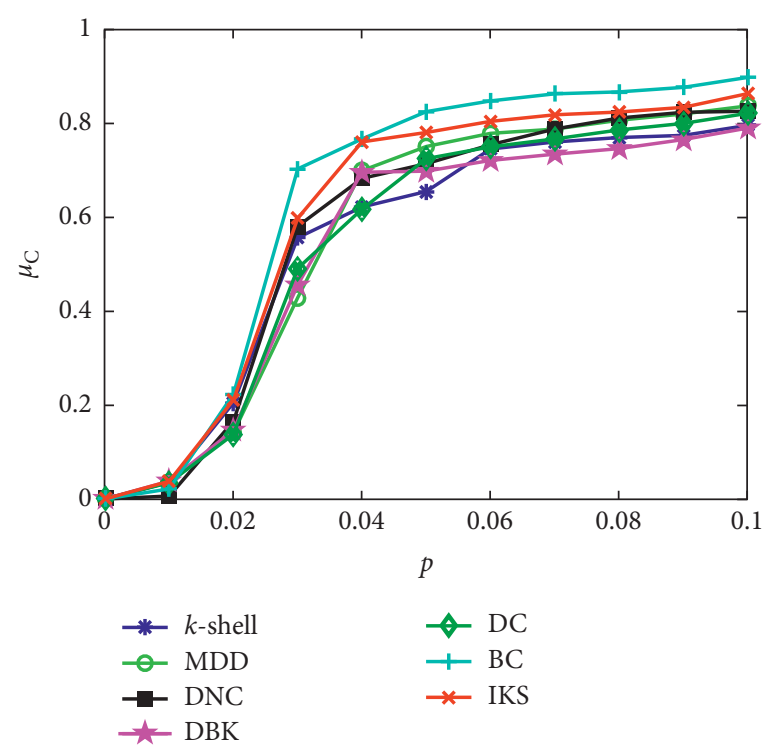

FIGURE 6: Relationship between the rate of decline of the network connectivity coefficient and the ratio of nodes removed.

in the network connectivity becomes more obvious, indicating that the removal of the node $v(i)$ is more influential.

Figure 6 compares and analyzes the ranking accuracy that is possible with the seven different methods regarding the influence of the nodes from the perspective of their network connectivity coefficient. This figure shows that when the first $10 \%$ of the influential nodes are removed using the BC method, the rate at which the network connectivity coefficient declines is the largest, followed by the IKS method. Based on the concept that "destructiveness is equal to importance," the BC method ranks the influences of the nodes most accurately. The IKS method is slightly worse than the BC method at ranking the influence of the nodes but outperforms the remaining five methods.

Figure 7 shows the change in the rate of decrease of the maximal connected subgraphs of the network with the proportion $p$ of the removed nodes, after the influential nodes identified by the different methods are removed. The rate of decrease $\mu_{\mathrm{S}}(i)$ of the maximum connected subgraph ratio also reflects the influence of the deleted node $v(i)$. The rate is calculated as follows:

$$
\mu_{\mathrm{S}}(i)=1-S_{i}^{\prime},
$$

where $S_{i}^{\prime}$ is the ratio of the maximal connected subgraphs of the network after removing the nodes. As the value of $\mu_{\mathrm{S}}(i)$ increases, the network is divided more uniformly, and the removed node $v(i)$ has a greater influence.

As can be seen from Figure 7, all the curves exhibit a downward trend as the proportion of removed nodes increases. Upon removing the first $1 \%$ of the influential nodes according to the sequences of the different methods, 


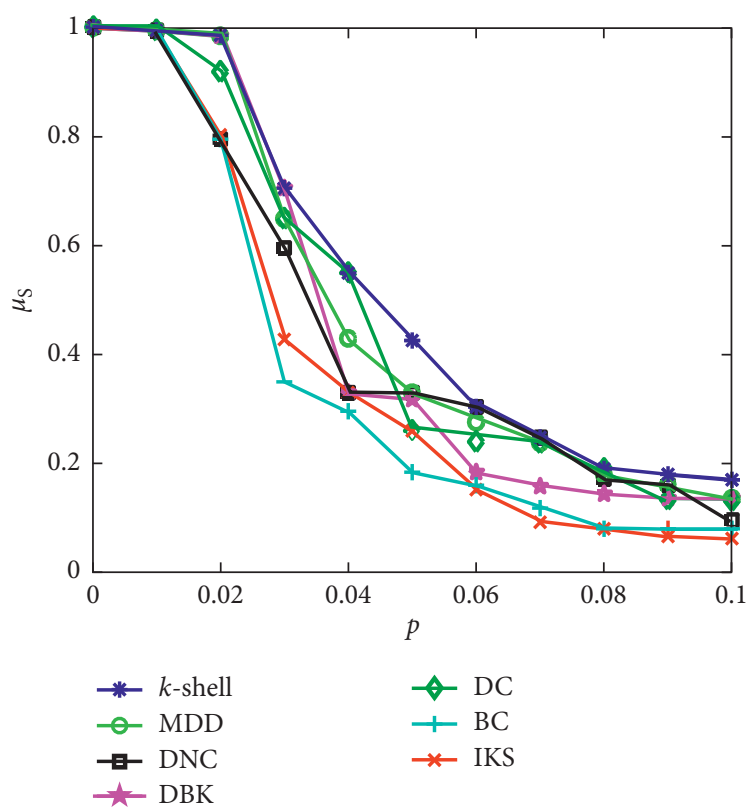

FIGURE 7: Relationship between the ratio of maximal connected subgraphs and the ratio of nodes removed.

the ratio of the maximal connected subgraphs of the network approaches 1 , and the curves of the different methods almost coincide, indicating that deleting very few influential nodes will not form a subnet. As the number of deleted influential nodes increases, the curve of the $\mathrm{BC}$ method decreases most rapidly, followed by that of the IKS method. The curves of the other methods are intertwined and difficult to distinguish. When the removal ratio of the influential nodes exceeds $6 \%$, the maximal connected subgraphs decrease most rapidly when the influential nodes are removed sequentially according to the IKS method.

\subsection{Impact of Influential Node Removal on Correlation} Analysis between the iks Value and Its Propagation Value. The present study used the SIR propagation model to simulate the propagation capability of the influential nodes. In the SIR model, individuals have three states: (1) susceptible state ( $S$ state), referring to the state of individual health, which may be infected as a result of contact with infected individuals; (2) infected state (I state), referring to the state of individual infection, which is infectious; and (3) removed/recovered state ( $\mathrm{R}$ state), which is that state in which an individual has either recovered from an infection and acquired immunity or died as a result of an infection, and thus can no longer affect other individuals, which is the equivalent of removal from the system. In the SIR model, nodes in the I state transmit an infectious disease to nodes in the $\mathrm{S}$ state with the probability $\alpha$. At the same time, a node in the I state passes through at time $\operatorname{Tr}$ after infection and recovers or dies with the probability $\beta$. The infection process and mechanism of the SIR model can be expressed as follows:

$$
\left\{\begin{array}{l}
S(i)+I(j) \stackrel{\alpha}{\longrightarrow} I(i)+I(j), \\
I(i) \stackrel{\beta}{\longrightarrow} R(i) .
\end{array}\right.
$$

For time $t$, the node densities in the S, I, and $\mathrm{R}$ states are $S(t), I(t)$, and $R(t)$, respectively, for which the propagation dynamics of the SIR method can be described as

$$
\left\{\begin{array}{l}
\frac{\mathrm{d} s(t)}{\mathrm{d} t}=-\alpha i(t) * s(t), \\
\frac{\mathrm{d} i(t)}{\mathrm{d} t}=\alpha i(t) * s(t)-\beta i(t), \\
\frac{\mathrm{d} r(t)}{\mathrm{d} t}=\beta i(t) .
\end{array}\right.
$$

When ranking the influence of the network nodes, the different methods start from the most influential node each time a node $v_{i}$ is designated as the source of infection. The SIR simulation of each node is carried out $N$ times. After time steps $T$, the number of non-S-state nodes $X_{i}$ is counted. In the present study, the average propagation value $\bar{X}_{i}$ and the standard deviation $\sigma(i)$ are used to measure the propagation ability of the node $v_{i}$. The potential propagation capability of a node can be expressed as

$$
\begin{aligned}
\overline{X_{i}} & =\frac{1}{N} \sum_{n=1}^{N} X_{i}(n), \\
\sigma(i) & =\sqrt{\frac{1}{N-1} \sum_{n=1}^{N}\left(X_{i}(n)-\overline{X_{i}}\right)^{2}},
\end{aligned}
$$




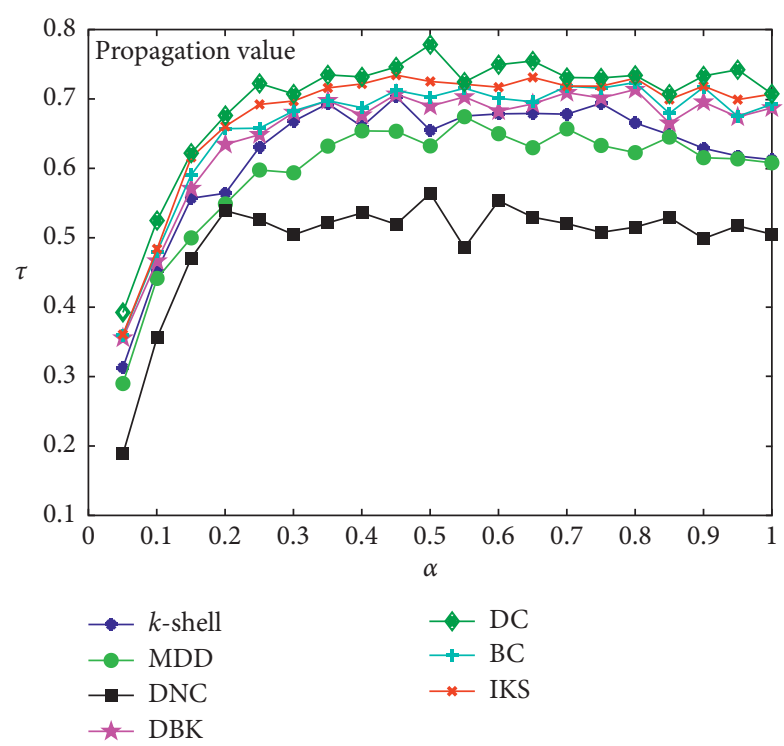

(a)

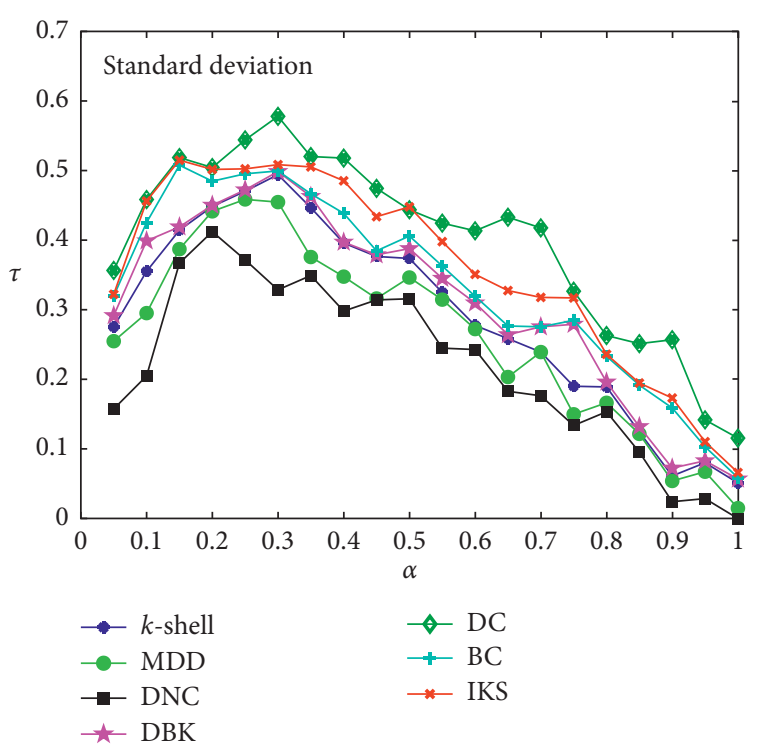

(b)

FIGURE 8: Relationship between the propagation value, standard deviation of influential nodes, and propagation rates. (a) Relation between the spreading value and transmission rates. (b) Relation between the standard deviation and transmission rates.

where $X_{i}(n)$ is the propagation value of the node $v_{i}$ in the $n^{\text {th }}$ simulation, that is, the number of nodes in the non-S state at the end of the $n^{\text {th }}$ simulation; $\overline{X_{i}}$ is the average propagation value after the $n^{\text {th }}$ simulation; and $\sigma(i)$ is the standard deviation of the propagation ability of the node $v_{i}$. The standard deviation reflects the deviation level of $X_{i}(n)$ and $\overline{X_{i}}$ and expresses the divergence or convergence of the propagation ability of the node $v_{i}$. As the value of $\sigma(i)$ increases, the difference in the transmission ability of the node $v_{i}$ also increases, as does the divergence of the transmission ability of the node $v_{i}$. Conversely, as the value of $\sigma(i)$ decreases, the transmission ability of the node $v_{i}$ becomes more convergent.

The simulation is based on the assumption that $\operatorname{Tr}=2$ and $\beta=1$; that is, the nodes in the I state change to the $\mathrm{R}$ state after $\operatorname{Tr}=2$ time steps, and each node is used as a source of infection according to the order of the nodes. After a certain time step $T$ (assumed to be 100 in the present study), the number of network nodes in the infected state is counted. Random errors are avoided by repeating the SIR simulation 100 times $(N=100)$ using each node as a source of infection.

Figure 8 shows the correlation between the propagation value, the standard deviation of the first 10\% of influential nodes, and the propagation rates for the seven methods as a function of the propagation rate $\alpha$. The correlation obtained for the DNC method is opposite to that of the influence of the nodes; that is, as the value of the influence decreases, the nodes become more influential. Therefore, the correlation coefficient between the influence of the nodes obtained using the DNC method and both its propagation value and standard deviation is negative. Thus, in Figure 8, the DNC curve was plotted using the absolute values of the results for convenience.
The relationship curve in Figure 8(a) shows that when the propagation rate $\alpha$ is small and increases gradually, the correlation between the propagation value and the influence value of the influential nodes also increases gradually. This indicates that the propagation ability of the influential nodes increases with the propagation rate. When the propagation rate $\alpha$ reaches a certain value $(\alpha \geq 0.4)$, the correlation coefficient between the propagation value and the influence value tends to be fixed. In comparison, the standard deviation of the node propagation value first increases and then decreases with the propagation rate. This proves that when the propagation rate reaches a certain level, the number of infected individuals in the network tends to be fixed, and the propagation values of the nodes tend to converge. An analysis of the amplitude of the curve reveals that the degree of the nodes best reflects the propagation ability of the nodes, followed by the IKS method, which is mainly related to the propagation mode of the virus in terms of "neighbor contact infection." At the same time, compared with other indicators, the IKS method can predict the propagation ability of nodes more accurately, which confirms the effectiveness of the proposed IKS algorithm. This is in good agreement with the conclusions of previous studies.

Similarly, Kendall's tau coefficients were used to compare the correlation between the node influence metrics obtained using the different methods and the node propagation ability. Subsequently, the ability of each method to accurately identify the influential nodes was analyzed. Figure 9 shows the correlation between the propagation capability of the nodes in a command and control network and the influence of the nodes obtained by various methods at a propagation rate $\alpha$ of 0.4 . Figures $9(\mathrm{a})-9(\mathrm{~g})$ show the 


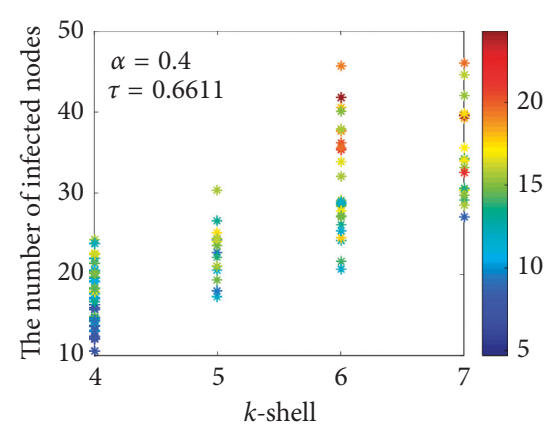

(a)

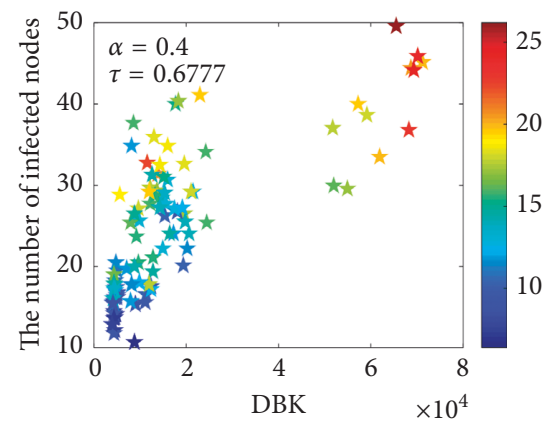

(d)

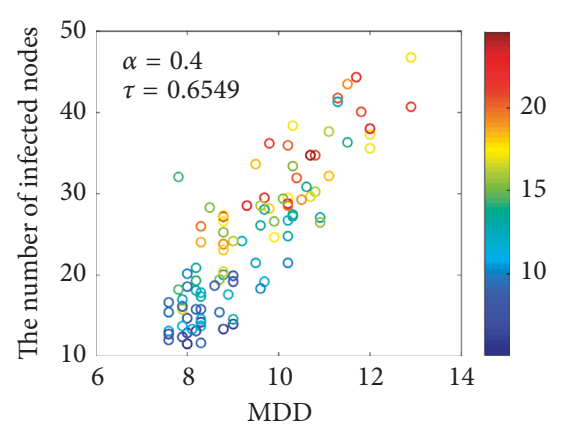

(b)

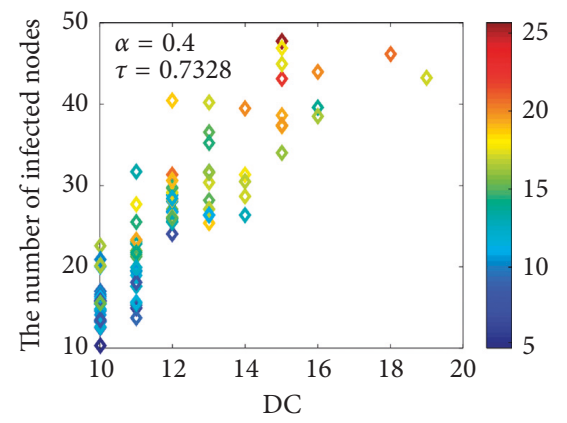

(e)

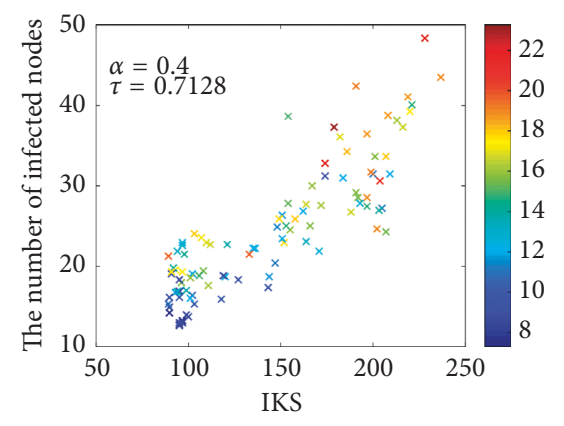

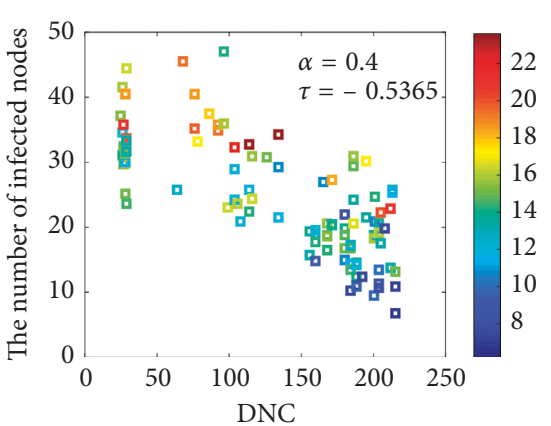

(c)

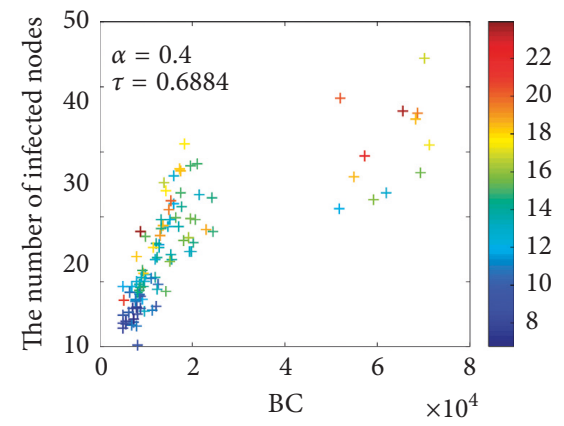

(f)

$(\mathrm{g})$

FIGURE 9: Correlation between the node influence value and the propagation value when the propagation rate $\alpha=0.4$.

TABLE 5: Comparison of performance of the seven influential node identification methods.

\begin{tabular}{lccc}
\hline Rank method & Network information & Computational complexity & Accuracy \\
\hline$k$-shell & Global information & $o(N)$ & Lower \\
MDD & Global information & $o(N)$ & Lower \\
DNC & Global information & $o\left(N^{2}\right)$ & Lower \\
DBK & Global information & $o\left(N^{3}\right)$ & Higher \\
DC & Local information & $o(N)$ & Lower \\
BC & Global information & $o\left(N^{3}\right)$ & Higher \\
IKS & Global and local information & $o\left(N^{2}\langle k\rangle\right)$ & Higher \\
\hline
\end{tabular}

relationship between the propagation capability and the importance of nodes, as obtained by the $k$-shell, MDD, DNC, DBK, DC, BC, and IKS algorithms, respectively. The circles in the graph represent the first $10 \%$ of the influential nodes obtained by each ranking method. The abscissa shows the influence value of the nodes under each ranking method, whereas the ordinate shows the propagation value of the corresponding nodes (the number of infected nodes in a network). The color indicates the standard deviation of the propagation value of the nodes.

As can be seen in Figure 9, the influence values of the nodes obtained using the six methods other than the DNC method are moderately correlated with the corresponding node propagation values $(\tau \in[0.3,0.8])$ for a propagation rate $\alpha$ of 0.4 . That is, as the influence values increase, the propagation ability of a node becomes 
stronger, and the standard deviation of the propagation values increases. The correlation coefficient between the integral $k$-shell index and the node propagation value obtained by the IKS method is 0.7128 , which is second only to the correlation coefficient between the degree index and the node propagation value. This shows that the IKS method can still rank the influence of the nodes in a command and control network accurately when the influence of the nodes is represented by their propagation ability.

Results based on a large number of simulations show that the integral $k$-shell method offers a higher degree of accuracy from the perspective of network destructiveness caused by the removal of the influential nodes. Although the performance of the integral $k$-shell method is slightly less accurate than the betweenness, the integral $k$-shell method has the advantage of low complexity. With respect to its node propagation ability, the integral $k$-shell method can also identify the influential nodes of the network more accurately. The network information, computational complexity, and accuracy required by the various methods are compared in Table 5.

Therefore, the proposed integral $k$-shell method has higher identification accuracy and lower computational complexity and can identify the influential nodes of the command and control network well.

\section{Conclusion}

This study addressed the problem of influential node identification in a command and control network. The study led to the proposal of a method to identify influential nodes in a command and control network based on an integral $k$ shell (IKS). The method was designed to overcome the problems associated with the existing $k$-shell method and improved methods based thereupon. The disadvantages of these methods are their coarse ranking granularity and the fact that they disregard the local centrality of the nodes, which lowers the accuracy of influential node identification. The proposed IKS method, which uses a refinement of the $k$ shell decomposition, was used to determine the influence of nodes by using the $k$-shell method and 2-order neighboring degree. The algorithm was described, and its computational complexity was deduced. The influence of node removal on the network performance and the correlation between the $i k s$ values of the nodes and their propagation values were compared and analyzed. The simulation results showed that the proposed IKS method has higher identification accuracy and lower computational complexity and confirmed its ability to efficiently identify the influential nodes of a command and control network. The key node identification IKS algorithm is applied to the SIR model and its improved virus propagation model, and its mathematical analysis is the next research focus.

\section{Data Availability}

The data used to support the findings of this study are available from the corresponding author upon request.

\section{Conflicts of Interest}

The authors declare that there are no conflicts of interest regarding the publication of this paper.

\section{Acknowledgments}

This work was supported by the Natural Science Foundation of China under Grant 61471080; the Equipment Development Department Research Foundation of China under Grant 61400010303; and the Natural Science Research Project of Liaoning Education Department of China under Grant JDL2019019.

\section{References}

[1] Y. M. Wang, S. Chen, C. S. Pan et al., "Measure of invulnerability for command and control network based on mission link," Information Sciences, vol. 426, pp. 148-159, 2018.

[2] D. A. Eisenberg, D. L. Alderson, M. Kitsak, A. Ganin, and I. Linkov, "Network foundation for command and control (C2) systems: literature review," IEEE Access, vol. 6, pp. 68782-68794, 2018.

[3] X. F. Hu, X. Y. He, and D. H. Rao, "A methodology for investigating the capabilities of command and coordination for system of system operation based on complex network theory," Complex Systems and Complexity Science, vol. 12, no. 2, pp. 9-17, 2015.

[4] X. Song, W. Shi, G. Tan, and Y. Ma, "Multi-level tolerance opinion dynamics in military command and control networks," Physica A: Statistical Mechanics and Its Applications, vol. 437, pp. 322-332, 2015.

[5] X. Gao, K. Li, and B. Chen, "Invulnerability measure of a military heterogeneous network based on network structure entropy," IEEE Access, vol. 6, pp. 6700-6708, 2018.

[6] X. F. Hu, "A brief survey on war complex networks studies," Complex Systems and Complexity Science, vol. 7, no. 2-3, pp. 24-28, 2010.

[7] A. H. Dekker, "Measuring the agility of networked military forces," Journal of Battlefield Technology, vol. 9, no. 1, pp. 19-24, 2006.

[8] R. Albert, H. Jeong, and A. L. Barabási, "Error and attack tolerance of complex networks," Nature, vol. 406, pp. 378-382, 2010.

[9] Y. P. Li, S. Y. Tan, Y. Deng et al., "Attacker-defender game from a network science perspective," CHAOS, vol. 28, no. 5, Article ID 051102, 2018.

[10] H. Yu, Z. Liu, and Y. J. Li, "Key nodes in complex networks identified by multi-attribute decision-making method," Acta Physica Sinica, vol. 62, no. 2, Article ID 020204, 2013.

[11] Z. Liu, C. Jiang, J. Wang, and H. Yu, "The node importance in actual complex networks based on a multi-attribute ranking method," Knowledge-Based Systems, vol. 84, pp. 56-66, 2015.

[12] D. Wei, X. Deng, X. Zhang, Y. Deng, and S. Mahadevan, "Identifying influential nodes in weighted networks based on evidence theory," Physica A: Statistical Mechanics and Its Applications, vol. 392, no. 10, pp. 2564-2575, 2013.

[13] T. Opsahl, F. Agneessens, and J. Skvoretz, "Node centrality in weighted networks: generalizing degree and shortest paths," Social Networks, vol. 32, no. 3, pp. 245-251, 2010. 
[14] U. Brandes, "A faster algorithm for betweenness centrality," The Journal of Mathematical Sociology, vol. 25, no. 2, pp. 163-177, 2001.

[15] Y. Li, W. G. Li, Y. Tan et al., "Hierarchical decomposition for betweenness centrality measure of complex networks," Scientific Reports, vol. 7, Article ID 46491, 2017.

[16] X. Q. Cheng, F. X. Ren, H. W. Shen et al., "Bridgeness: a local index on edge significance in maintaining global connectivity," Journal of Statistical Mechanics: Theory and Experiment, vol. 2010, no. 10, Article ID P10011, 2010.

[17] R. Poulin, M.-C. Boily, and B. R. Mâsse, "Dynamical systems to define centrality in social networks," Social Networks, vol. 22, no. 3, pp. 187-220, 2000.

[18] J. Pijitrai, S. Siripun, and C. Worasit, "CiteRank: Combination similarity and static ranking with research paper searching," International Journal of Internet Technology \& Secured Transaction, vol. 3, no. 2, pp. 161-177, 2011.

[19] R. Criado, S. Moral, A. Perez et al., "On the edges' PageRank and line graphs," CHAOS, vol. 28, no. 7, Article ID 075503, 2018.

[20] Q. Li, T. Zhou, L. Lü, and D. Chen, "Identifying influential spreaders by weighted leaderrank," Physica A: Statistical Mechanics and Its Applications, vol. 404, pp. 47-55, 2014.

[21] L. L. Jiang, M. Medo, J. R. Wakeling et al., "Building reputation systems for better ranking," 2010, https://arxiv.org/abs/ 1001.2186.

[22] X. P. Su and Y. R. Song, "Leveraging neighborhood "structural holes" to identifying key spreaders in social networks," Acta Physica Sinica, vol. 64, no. 2, Article ID 020101, 2015.

[23] Y. M. Wang, Q. Y. Wang, C. S. Pan et al., "Method for key nodes identification in command and control network by considering structural holes," Fire Control and Command Control, vol. 42, no. 3, pp. 59-63, 2017.

[24] M. Kitsak, L. K. Gallos, S. Havlin et al., "Identification of influential spreaders in complex networks," Nature Physics, vol. 6, no. 11, pp. 888-893, 2010.

[25] D. B. Chen, H. Gao, L. Y. Lü et al., "Identifying influential nodes in large-scale directed networks: the role of clustering," PLoS One, vol. 8, no. 10, Article ID e77455, 2013.

[26] D. Chen, L. Lü, M.-S. Shang, Y.-C. Zhang, and T. Zhou, "Identifying influential nodes in complex networks," Physica A: Statistical Mechanics and Its Applications, vol. 391, no. 4, pp. 1777-1787, 2012.

[27] A. Zeng and C.-J. Zhang, "Ranking spreaders by decomposing complex networks," Physics Letters A, vol. 377, no. 14, pp. 1031-1035, 2013.

[28] J.-G. Liu, Z.-M. Ren, and Q. Guo, "Ranking the spreading influence in complex networks," Physica A: Statistical Mechanics and Its Applications, vol. 392, no. 18, pp. 4154-4159, 2013.

[29] Z. M. Ren, J. G. Liu, F. Shao et al., "Analysis of the spreading influence of the nodes with minimum $k$-shell value in complex networks," Acta Physica Sinica, vol. 62, no. 10, Article ID 108902, 2013.

[30] B. Hou, Y. Yao, and D. Liao, "Identifying all-around nodes for spreading dynamics in complex networks," Physica A: Statistical Mechanics and Its Applications, vol. 391, no. 15, pp. 4012-4017, 2012.

[31] D. Helbing, D. Brockmann, T. Chadefaux et al., "Saving human lives: what complexity science and information systems can contribute," Journal of Statistical Physics, vol. 158, no. 3, pp. 735-781, 2015.
[32] B. Podobnik, D. Horvatic, T. Lipic et al., "The cost of attack in competing networks," Journal of the Royal Society Interface, vol. 12, no. 112, Article ID 20150770, 2015.

[33] M. Jalili and M. Perc, "Information cascades in complex networks," Journal of Complex Networks, vol. 5, no. 5, pp. 665-693, 2017.

[34] M. Perc, "The Matthew effect in empirical data," Journal of the Royal Society Interface, vol. 11, no. 98, Article ID 20140378, 2014.

[35] J. Wu, M. Barahona, Y. J. Tan et al., "Robustness of random graphs based on graph spectra," CHAOS, vol. 22, no. 4, Article ID 043101, 2012.

[36] J. C. Li, J. Wu, Y. J. Tan et al., "Robustness of combat networks based on directed natural connectivity," Complex Systems and Complexity Science, vol. 12, no. 4, pp. 25-30, 2015.

[37] G.-S. Peng and J. Wu, "Optimal network topology for structural robustness based on natural connectivity," Physica A: Statistical Mechanics and Its Applications, vol. 443, pp. 212-220, 2016.

[38] O. Lordan, J. M. Sallan, P. Simo, and D. Gonzalez-Prieto, "Robustness of airline alliance route networks," Communications in Nonlinear Science Numerical Simulation, vol. 22, no. 1-3, pp. 587-595, 2015.

[39] C. Deng, "The robustness analysis of wireless sensor networks under uncertain interference," Scientific World Journal, vol. 2013, Article ID 185970, 8 pages, 2013.

[40] X. He and Y. Wu, "Affects of production adjustment strategy between manufactures on supply chain stability," Science and Technology Management Research, vol. 32, no. 21, pp. 225228, 2012.

[41] C. Yen, M. Y. Yeh, and M. Chen, “An efficient approach to updating closeness centrality and average path length in dynamic networks," in Proceedings of the IEEE 13st International Conference on Data Mining, pp. 867-876, Dallas, TX, USA, December 2013.

[42] J. R. Cares, "An information age combat model," in Proceedings of the 9th International Command and Control Research and Technology Symposium, Copenhagen, Denmark, September 2004.

[43] S. Deller, M. I. Bell, S. R. Bowling et al., "Applying the information age combat model: quantitative analysis of network centric operations," International C2 Journal, vol. 3, no. 1, pp. 1-25, 2009.

[44] S. Deller, G. Rabadi, A. Tolk, and S. R. Bowling, "Organizing for improved effectiveness in networked operations," Military Operations Research, vol. 17, no. 1, pp. 5-16, 2012.

[45] A. R. Benson, D. F. Gleich, and J. Leskovec, "Higher-order organization of complex networks," Science, vol. 353, no. 6295, pp. 163-166, 2016.

[46] L. Y. Lue, T. Zhou, Q. M. Zhang et al., "The H-index of a network node and its relation to degree and coreness," Nature Communications, vol. 7, Article ID 10168, 2016.

[47] Y.-L. Lee and T. Zhou, "Fast asynchronous updating algorithms for $k$-shell indices," Physica A: Statistical Mechanics and Its Applications, vol. 482, pp. 524-531, 2017.

[48] X. E. Gao, D. P. Zhang, K. Q. Li et al., "A cascading failure model for command and control networks with hierarchy structure," Security and Communication Networks, vol. 2018, Article ID 6063837, 14 pages, 2018. 


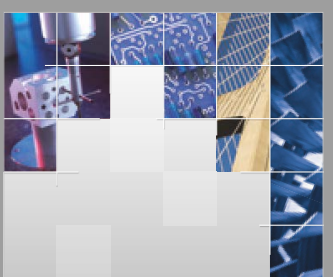

\section{Enfincering}
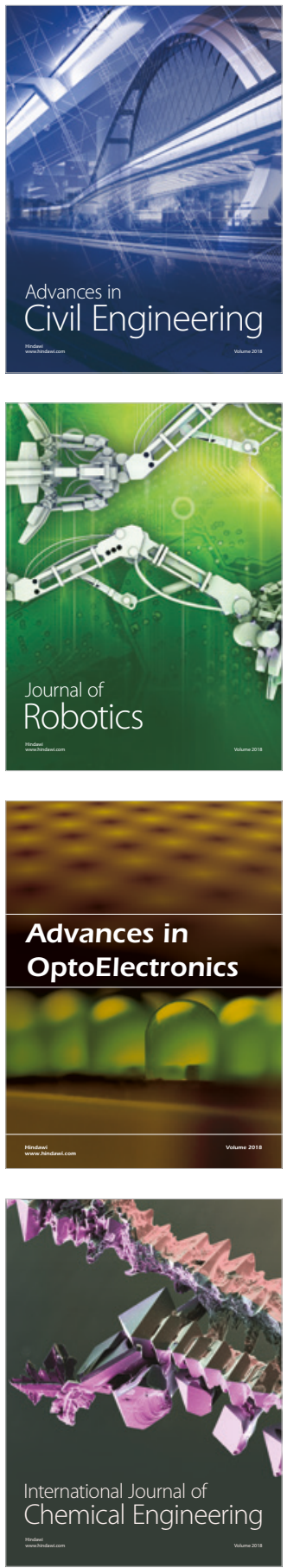

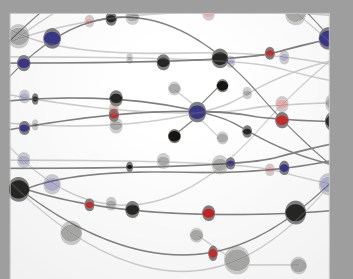

\section{Rotating \\ Machinery}

The Scientific World Journal

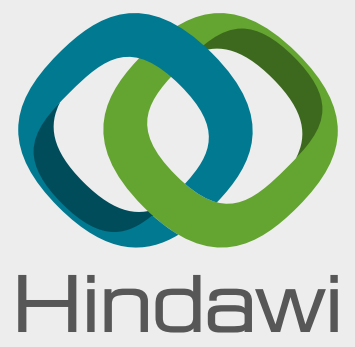

Submit your manuscripts at

www.hindawi.com
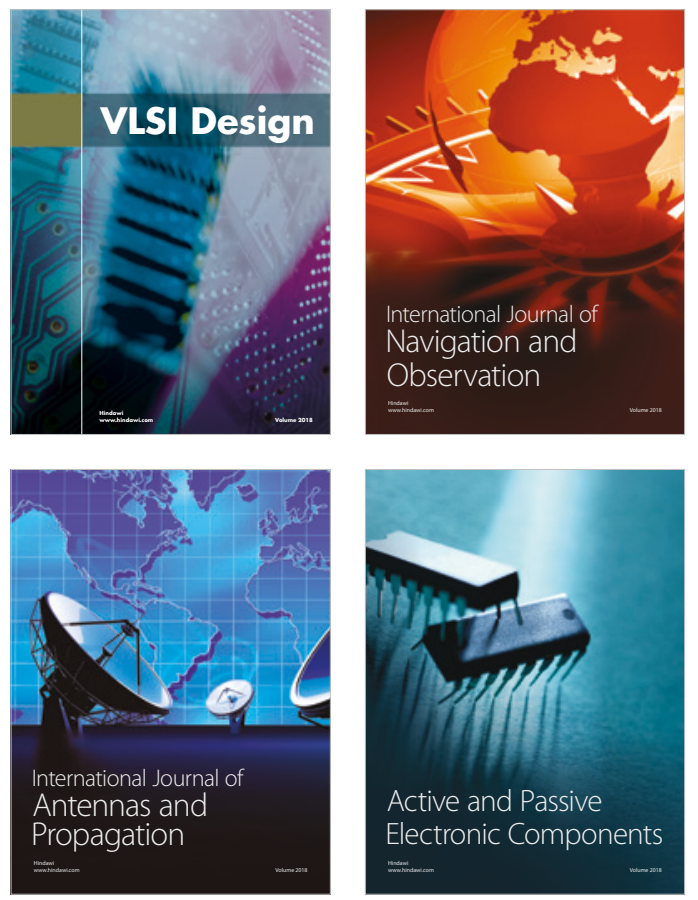
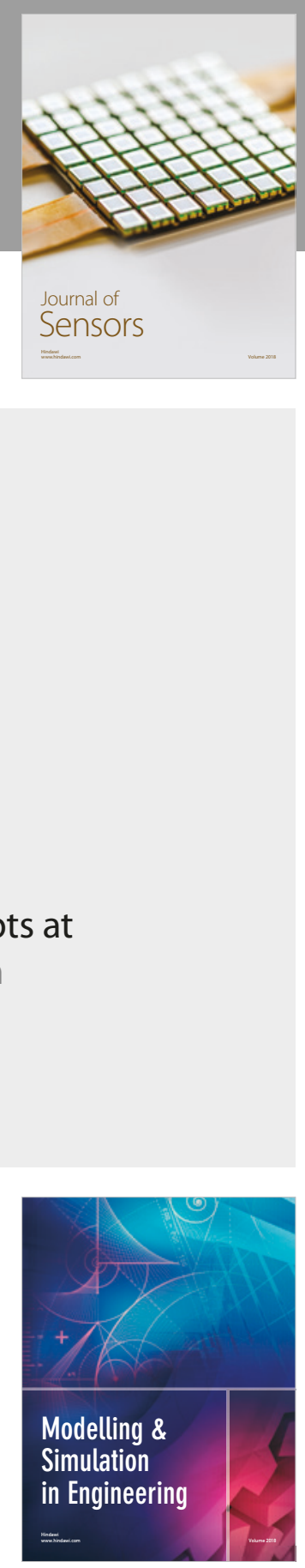

\section{Advances \\ Multimedia}
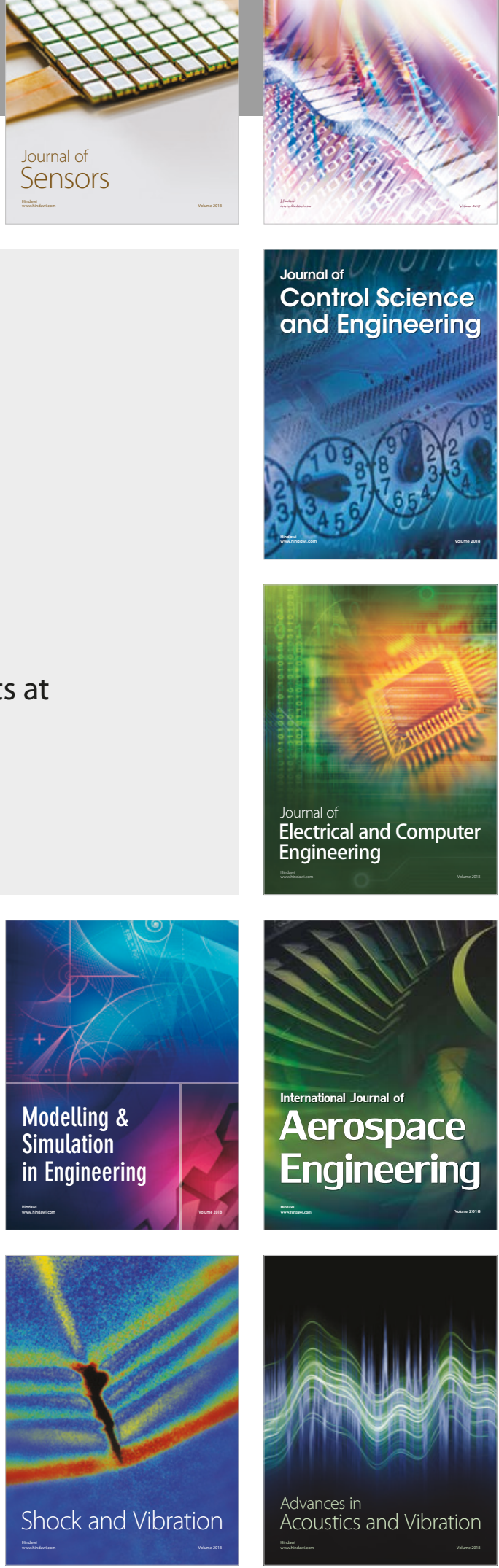